\title{
Exact small deviation asymptotics for some Brownian functionals
}

\author{
Ruslan Pusev \\ (joint work with Yakov Nikitin)
}

Saint-Petersburg State University

Huntsville, June 7, 2012 


\section{Outline}

(1) Small deviations in the weighted quadratic norm

(2) Small deviations of the Brownian excursion

(3) Small deviations of the Brownian meander

(4) Small deviations of the Brownian local time 


\section{$L_{2}$-small ball probabilities}

Let $X(t), 0 \leq t \leq 1$, be a zero mean Gaussian process with covariance function $G(t, s)=\mathrm{E} X(t) X(s)$ and let $\psi$ be a nonnegative function on $[0,1]$. Denote

$$
\|X\|_{\psi}=\left(\int_{0}^{1} X^{2}(t) \psi(t) d t\right)^{1 / 2} .
$$

The problem is to describe the behavior of $\mathrm{P}\left\{\|X\|_{\psi} \leq \varepsilon\right\}$ as $\varepsilon \rightarrow 0$. 


\section{$L_{2}$-small ball probabilities}

The problem was solved by Sytaya (1974), but in an implicit way. Later Zolotarev; Dudley, Hoffmann-Jørgensen and Shepp; Ibragimov; W. Li; Dunker, Lifshits and Linde; Gao, Hannig, Lee and Torcaso; Nazarov and Nikitin, etc. improved and simplified the expression for $\mathrm{P}\left\{\|X\|_{\psi} \leq \varepsilon\right\}$. 


\section{Karhunen-Loève expansion}

Karhunen-Loève expansion implies

$$
\|X\|_{\psi}^{2}=\sum_{k=1}^{\infty} \lambda_{k} \xi_{k}^{2}
$$

where $\xi_{k}$ are i.i.d. $N(0,1)$ r.v.'s,

$\lambda_{k}>0$ are the eigenvalues of the integral equation

$$
\lambda f(t)=\int_{0}^{1} G(t, s) \sqrt{\psi(t) \psi(s)} f(s) d s, \quad t \in[0,1] .
$$




\section{Comparison theorem}

W. Li, 1992:

Comparison theorem. Let $\xi_{k}$ be i.i.d. $N(0,1)$ r.v.'s, and let $a_{k}>0$ and $b_{k}>0$ be such that $\sum_{k=1}^{\infty}\left|1-a_{k} / b_{k}\right|<\infty$. Then, as $\varepsilon \rightarrow 0$,

$$
\mathrm{P}\left\{\sum_{k=1}^{\infty} a_{k} \xi_{k}^{2} \leq \varepsilon^{2}\right\} \sim\left(\prod_{k=1}^{\infty} \frac{b_{k}}{a_{k}}\right)^{1 / 2} \mathrm{P}\left\{\sum_{k=1}^{\infty} b_{k} \xi_{k}^{2} \leq \varepsilon^{2}\right\} .
$$

F. Gao, J. Hannig, F. Torcaso, 2003:

$\sum_{k=1}^{\infty}\left|1-a_{k} / b_{k}\right|<\infty$ was replaced by $0<\prod_{k=1}^{\infty}\left(a_{k} / b_{k}\right)<\infty$. 


\section{Exact asymptotics}

In typical cases the exact asymptotics has the form

$$
\begin{gathered}
\mathrm{P}\left\{\|X\|_{\psi} \leq \varepsilon\right\} \sim C \varepsilon^{\gamma} \exp \left(-K \varepsilon^{-\alpha}\right) . \\
\text { If } \lambda_{k}=\left(\vartheta\left(k+\delta+O\left(k^{-1}\right)\right)\right)^{-d} \text {, then } \\
\alpha=\alpha(d), \quad K=K(d, \vartheta), \\
\gamma=\gamma(d, \delta), \quad C=C(\vartheta, d, \delta) \cdot C_{\text {dist }}\left(\lambda_{k}, k \in \mathbb{N}\right) .
\end{gathered}
$$




\section{Exact asymptotics}

Here

$$
C_{\text {dist }}=\left(\prod_{k=1}^{\infty} \frac{(\vartheta(k+\delta))^{-d}}{\lambda_{k}}\right)^{1 / 2}
$$

When the eigenfunctions of the covariance can be expressed in terms of elementary or special functions, there exist explicit formulas for the distortion constants (A. Nazarov, 2003, A. Nazarov, R. Pusev, 2009). 


\section{Previous results for Wiener process}

A. Nazarov, R. Pusev, 2009:

$\mathrm{P}\left\{\int_{0}^{1} \frac{W^{2}(t)}{\left(a^{2}+t^{2}\right)^{2}} d t \leqslant \varepsilon^{2}\right\} \sim \frac{4 a^{1 / 2}\left(1+a^{2}\right)^{1 / 4}}{\pi^{1 / 2} \operatorname{arcctg} a} \varepsilon \exp \left(-\frac{(\operatorname{arcctg} a)^{2}}{8 a^{2} \varepsilon^{2}}\right)$,

$\mathrm{P}\left\{\int_{0}^{1} \frac{W^{2}(t)}{\left(a^{2}-t^{2}\right)^{2}} d t \leqslant \varepsilon^{2}\right\} \sim \frac{8\left(a^{2}-1\right)^{1 / 4} a^{1 / 2}}{\pi^{1 / 2} \ln \frac{a+1}{a-1}} \varepsilon \exp \left(-\frac{\left(\ln \frac{a+1}{a-1}\right)^{2}}{32 a^{2} \varepsilon^{2}}\right)$

$P\left\{\int_{0}^{1}(t+a)^{2 \beta-2} W^{2}(t) d t \leqslant \varepsilon^{2}\right\} \sim$

$$
\sim \frac{\left(a(a+1)^{-1}\right)^{(\beta-1) / 4} \beta}{\left((a+1)^{\beta}-a^{\beta}\right)} \frac{4 \varepsilon}{\sqrt{\pi}} \exp \left(-\frac{\left((a+1)^{\beta}-a^{\beta}\right)^{2}}{8 \beta^{2} \varepsilon^{2}}\right) .
$$




\section{Previous results for Wiener process}

For

$$
\begin{array}{cc}
\psi(t)=\left(a^{2}+t^{2}\right)^{-2}, & a>0, \\
\psi(t)=\left(a^{2}-t^{2}\right)^{-2}, & a>1, \\
\psi(t)=(t+a)^{\beta}, & a>0,
\end{array}
$$

one has

$$
\mathrm{P}\left\{\|W\|_{\psi} \leqslant \varepsilon\right\} \sim \frac{4 \psi^{1 / 8}(0)}{\sqrt{\pi} \vartheta \psi^{1 / 8}(1)} \varepsilon \exp \left(-\frac{\vartheta^{2}}{8} \varepsilon^{-2}\right),
$$

where

$$
\vartheta=\int_{0}^{1} \sqrt{\psi(t)} d t
$$



Small deviations of the Brownian local time

\section{Question}

Are these relations special or typical? 


\section{Regular-shaped weights}

We show how to evaluate the distortion constant for some Gaussian processes when the eigenfunctions of the covariance are unknown. 


\section{Wiener process with regular-shaped weight}

Theorem 1. Let the weight $\psi$, defined on $[0,1]$, be positive and twice continuously differentiable. Put $\vartheta=\int_{0}^{1} \sqrt{\psi(t)} d t$. Then as $\varepsilon \rightarrow 0$

$$
\mathrm{P}\left\{\|W\|_{\psi} \leqslant \varepsilon\right\} \sim \frac{4 \psi^{1 / 8}(0)}{\sqrt{\pi} \vartheta \psi^{1 / 8}(1)} \varepsilon \exp \left(-\frac{\vartheta^{2}}{8} \varepsilon^{-2}\right) .
$$

We shall call the weight $\psi$ satisfying the conditions of this Theorem regular-shaped. 


\section{Sketch of proof}

The coefficients $\lambda_{k}$ in the $\mathrm{KL}$ expansion satisfy $\lambda_{k}=\mu_{k}^{-1}$, where $\mu_{k}$ are the eigenvalues of the BVP

$$
\left\{\begin{array}{l}
-y^{\prime \prime}=\mu \psi y \quad \text { on } \quad[0,1] \\
y(0)=y^{\prime}(1)=0
\end{array}\right.
$$

We have

$$
\mu_{k}=\left(\pi(k-1 / 2) / \vartheta+O\left(k^{-1}\right)\right)^{2},
$$

and

$$
\mathrm{P}\left\{\|W\|_{\psi} \leq \varepsilon\right\} \sim C_{\text {dist }} \mathrm{P}\left\{\sum_{k=1}^{\infty} \frac{\xi_{k}^{2}}{(\pi(k-1 / 2))^{2}} \leq\left(\frac{\varepsilon}{\vartheta}\right)^{2}\right\} .
$$




\section{Sketch of proof}

Denote by $\varphi_{1,2}(t, \zeta)$ the solutions of the equation $-y^{\prime \prime}=\zeta^{2} \psi y$ satisfying the initial conditions

$$
\begin{array}{ll}
\varphi_{1}(0, \zeta)=1, & \varphi_{1}^{\prime}(0, \zeta)=0, \\
\varphi_{2}(0, \zeta)=0, & \varphi_{2}^{\prime}(0, \zeta)=1 .
\end{array}
$$

We have $\mu_{k}=x_{k}^{2}$, where $x_{1}<x_{2}<\ldots$ are the positive roots of the function

$$
F(\zeta)=\operatorname{det}\left[\begin{array}{ll}
\varphi_{1}(0, \zeta) & \varphi_{2}(0, \zeta) \\
\varphi_{1}^{\prime}(1, \zeta) & \varphi_{2}^{\prime}(1, \zeta)
\end{array}\right]=\varphi_{2}^{\prime}(1, \zeta)
$$




\section{Sketch of proof}

Using WKB approximation, we obtain

$$
\varphi_{2}^{\prime}(1, \zeta)=\frac{\psi^{1 / 4}(1) \cos (\vartheta \zeta)}{\psi^{1 / 4}(0)}\left(1+O\left(\zeta^{-1}\right)\right) .
$$

Applying Jensen's theorem to the functions $F(\zeta)$ and $\cos (\vartheta \zeta)$, we get

$$
C_{\text {dist }}^{2}=\frac{\psi^{1 / 4}(0)}{\psi^{1 / 4}(1)} .
$$




\section{Brownian bridge with regular-shaped weight}

Let $B$ be the Brownian bridge.

Theorem 2. Let $\psi$ be a regular-shaped weight on $[0,1]$. Then as $\varepsilon \rightarrow 0$

$$
\mathrm{P}\left\{\|B\|_{\psi} \leq \varepsilon\right\} \sim \frac{2 \sqrt{2} \psi^{1 / 8}(0) \psi^{1 / 8}(1)}{\sqrt{\pi \vartheta}} \exp \left(-\frac{\vartheta^{2}}{8} \varepsilon^{-2}\right) .
$$




\section{Ornstein-Uhlenbeck process with regular-shaped weight}

Denote by $U_{(\alpha)}(t)$ a usual stationary Ornstein-Uhlenbeck process, that is, a centered Gaussian process with covariance

$$
G_{U_{(\alpha)}}(t, s)=e^{-\alpha|t-s|} /(2 \alpha) .
$$

Theorem 3. Let $\psi$ be a regular-shaped weight on $[0,1]$. Then as $\varepsilon \rightarrow 0$

$$
\mathrm{P}\left\{\left\|U_{(\alpha)}\right\|_{\psi} \leq \varepsilon\right\} \sim \frac{8 \alpha^{1 / 2} e^{\alpha / 2}}{\pi^{1 / 2} \vartheta^{3 / 2} \psi^{1 / 8}(0) \psi^{1 / 8}(1)} \varepsilon^{2} \exp \left(-\frac{\vartheta^{2}}{8} \varepsilon^{-2}\right) .
$$




\section{Ornstein-Uhlenbeck process with regular-shaped weight}

Denote by $\stackrel{\cup}{(\alpha)}_{(}(t)$ the Ornstein-Uhlenbeck process starting at zero, that is, the centered Gaussian process with the covariance function

$$
G_{\dot{U}_{(\alpha)}}(t, s)=\left(e^{-\alpha|t-s|}-e^{-\alpha(t+s)}\right) /(2 \alpha) .
$$

Theorem 4. Let $\psi$ be a regular-shaped weight on $[0,1]$. Then as $\varepsilon \rightarrow 0$

$$
\mathrm{P}\left\{\| \stackrel{\cup}{(\alpha)}_{(\alpha)} \leq \varepsilon\right\} \sim \frac{4 e^{\alpha / 2} \psi^{1 / 8}(0)}{\sqrt{\pi} \vartheta \psi^{1 / 8}(1)} \varepsilon \exp \left(-\frac{\vartheta^{2}}{8} \varepsilon^{-2}\right) .
$$




\section{Bogoliubov process with regular-shaped weight}

Denote by $Y(t)$ the Bogoliubov process, that is, the centered Gaussian process with covariance

$$
G_{Y}(t, s)=\frac{1}{2 \omega \sinh (\omega / 2)} \cosh \left(\omega|t-s|-\frac{\omega}{2}\right), \quad \omega>0 .
$$

Theorem 5. Let $\psi$ be a regular-shaped weight on $[0,1]$. Then as $\varepsilon \rightarrow 0$

$$
\mathrm{P}\left\{\|Y\|_{\psi} \leq \varepsilon\right\} \sim \frac{8 \sinh (\omega / 2) \psi^{1 / 8}(0) \psi^{1 / 8}(1)}{\vartheta \pi^{1 / 2}\left(\psi^{1 / 2}(0)+\psi^{1 / 2}(1)\right)^{1 / 2}} \varepsilon \exp \left(-\frac{\vartheta^{2}}{8} \varepsilon^{-2}\right) .
$$




\section{Non-Gaussian processes}

For a non-Gaussian process $X(t)$ the use of random series of Karhunen-Loève-type becomes problematic. The problem simplifies if the process $X(t)$ can be expressed by means of simple Gaussian processes. 


\section{Brownian excursion}

Denote by $\mathfrak{e}(t), 0 \leq t \leq 1$, a Brownian excursion.

We may imagine Brownian excursion on $[0,1]$ as the Brownian motion starting at zero, conditioned to stay positive and to hit zero for the first time at time 1 .

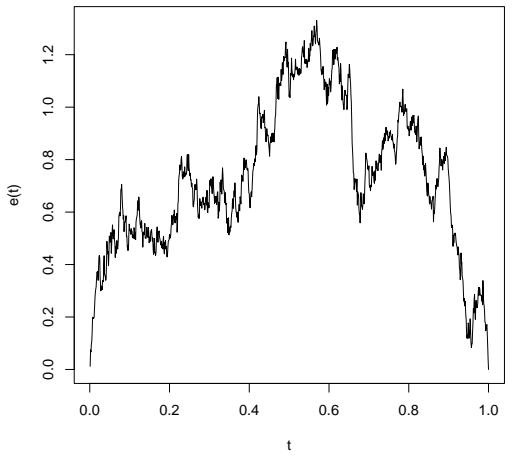




\section{Brownian excursion}

According to the Lévy-Williams identity,

$$
\left\{\mathfrak{e}^{2}(t), 0 \leq t \leq 1\right\} \stackrel{\text { law }}{=}\left\{B_{1}^{2}(t)+B_{2}^{2}(t)+B_{3}^{2}(t), 0 \leq t \leq 1\right\},
$$

where $B_{1}(t), B_{2}(t), B_{3}(t), 0 \leq t \leq 1$, are three independent Brownian bridges. Hence,

$$
\|\mathfrak{e}\|_{\psi}^{2} \stackrel{\text { law }}{=}\left\|B_{1}\right\|_{\psi}^{2}+\left\|B_{2}\right\|_{\psi}^{2}+\left\|B_{3}\right\|_{\psi}^{2} .
$$




\section{Brownian excursion}

Theorem 6. Let $\psi$ be a regular-shaped weight on $[0,1]$. Then as $\varepsilon \rightarrow 0$

$$
\mathrm{P}\left\{\|\mathfrak{e}\|_{\psi} \leq \varepsilon\right\} \sim \frac{2 \sqrt{6 \vartheta} \psi^{3 / 8}(0) \psi^{3 / 8}(1)}{\sqrt{\pi}} \varepsilon^{-2} \exp \left(-\frac{9 \vartheta^{2}}{8} \varepsilon^{-2}\right) .
$$

This result is new even for the unit weight $\psi \equiv 1$ ! 


\section{Brownian excursion}

Theorem 7. As $\varepsilon \rightarrow 0$

$$
\mathrm{P}\left\{\int_{0}^{1} \frac{\mathfrak{e}^{2}(t)}{t(1-t)} d t \leq \varepsilon^{2}\right\} \sim \frac{9 \pi^{3}}{\varepsilon^{5}} \exp \left(-\frac{9 \pi^{2}}{8 \varepsilon^{2}}\right) .
$$

Let $\beta>-2$. Then as $\varepsilon \rightarrow 0$

$$
\begin{aligned}
\mathrm{P}\left\{\int_{0}^{1} t^{\beta} \mathfrak{e}^{2}(t) d t\right. & \left.\leq \varepsilon^{2}\right\} \sim \frac{4 \pi^{1 / 4}}{3^{(\beta-4) /(4(\beta+2))} \Gamma^{3 / 2}\left(\frac{\beta+3}{\beta+2}\right)} \times \\
& \times((\beta+2) \varepsilon)^{-\frac{\beta+8}{2(\beta+2)}} \exp \left(-\frac{9}{2}((\beta+2) \varepsilon)^{-2}\right) .
\end{aligned}
$$




\section{Brownian excursion}

Denote by Wat $(\mathfrak{e})$ the Watson-type functional of the Brownian excursion:

$$
\operatorname{Wat}(\mathfrak{e})=\int_{0}^{1}\left(\mathfrak{e}(t)-\int_{0}^{1} \mathfrak{e}(x) d x\right)^{2} d t .
$$

Theorem 8. As $\varepsilon \rightarrow 0$

$$
\mathrm{P}\left\{\operatorname{Wat}(\mathfrak{e}) \leq \varepsilon^{2}\right\} \sim \sqrt{\frac{2}{\pi}} \varepsilon^{-1} \exp \left(-\frac{1}{8 \varepsilon^{2}}\right) .
$$




\section{Brownian meander}

Denote by $\mathfrak{m}^{z}(t), 0 \leq t \leq 1$, a Brownian meander taking the value $z \geq 0$ at the point 1 .

We may think of $\mathfrak{m}^{z}(t)$ as the Brownian motion starting at zero, conditioned to stay positive and to take the value $z$ at time 1 .

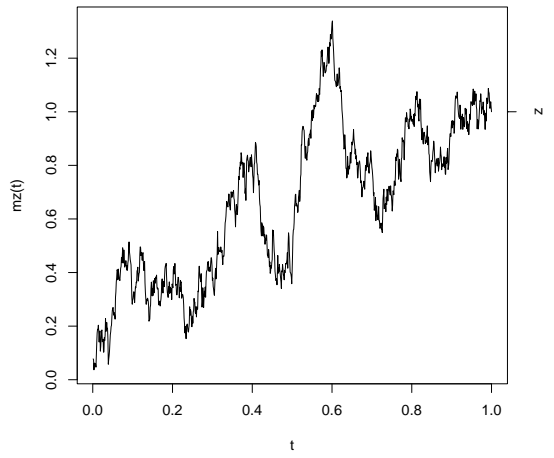




\section{Brownian meander}

For the Brownian meander taking the value $z \geq 0$ at the point 1 , one has (J. Bertoin, J. Pitman, J. Ruiz de Chavez, 1999)

$$
\left(\mathfrak{m}^{z}(t)\right)^{2} \stackrel{\text { law }}{=} B_{1}^{2}(t)+B_{2}^{2}(t)+\left(B_{3}(t)+z t\right)^{2}, 0 \leq t \leq 1,
$$

where $B_{1}, B_{2}$, and $B_{3}$ are three independent Brownian bridges.

Theorem 9. For any $z \geq 0$, we have as $\varepsilon \rightarrow 0$

$$
\mathrm{P}\left\{\left\|\mathfrak{m}^{z}\right\| \leq \varepsilon\right\} \sim \frac{2 \sqrt{2\left(z^{2}+3\right)}}{\sqrt{\pi}} \varepsilon^{-2} \exp \left(-\frac{\left(z^{2}+3\right)^{2}}{8} \varepsilon^{-2}+\frac{z^{2}}{2}\right) .
$$




\section{Brownian meander}

Denote by $\mathfrak{m}(t), 0 \leq t \leq 1$, a Brownian meander.

The Brownian meander can be thought of as a Brownian motion conditioned to stay positive up to time 1 ,

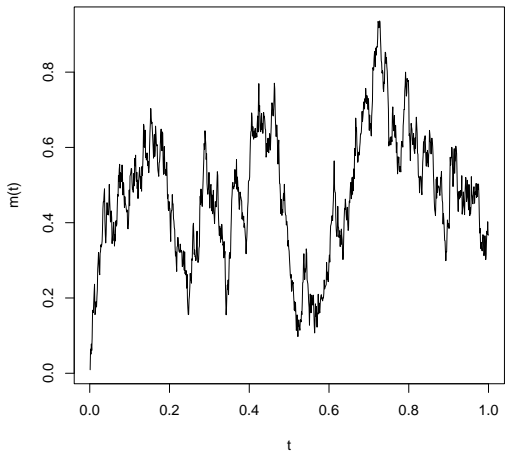




\section{Brownian meander}

J. Pitman, M. Yor, 1996: for the Brownian meander $\mathfrak{m}$, one has

$$
\left\{\mathfrak{m}^{2}(t), 0 \leq t \leq 1\right\} \stackrel{\text { law }}{=}\left\{B^{2}(t)+W_{1}^{2}(t)+W_{2}^{2}(t), 0 \leq t \leq 1\right\},
$$

where the Brownian bridge $B$ and two Brownian motions $W_{1}$ and $W_{2}$ are independent.

Theorem 10. For a regular-shaped weight function $\psi$ on $[0,1]$, we have

$$
\mathrm{P}\left\{\|\mathfrak{m}\|_{\psi} \leq \varepsilon\right\} \sim 4 \sqrt{\frac{2}{3 \pi}} \frac{\psi^{3 / 8}(0)}{\vartheta^{1 / 2} \psi^{1 / 8}(1)} \exp \left(-\frac{9 \vartheta^{2}}{8} \varepsilon^{-2}\right) .
$$


Small deviations in the weighted quadratic norm Small deviations of the Brownian excursion Small deviations of the Brownian meander Small deviations of the Brownian local time

\section{Brownian meander}

Theorem 11. Let $\beta>-2$. As $\varepsilon \rightarrow 0$

$$
\begin{aligned}
\mathrm{P}\left\{\int_{0}^{1} t^{\beta} \mathfrak{m}^{2}(t) d t\right. & \left.\leq \varepsilon^{2}\right\} \sim \frac{2^{2+\frac{\beta}{2(\beta+2)}} \pi^{\frac{1}{2}}}{3^{\frac{1}{2}+\frac{3 \beta}{4(\beta+2)}} \Gamma\left(\frac{1}{\beta+2}\right) \Gamma 1 / 2\left(\frac{\beta+3}{\beta+2}\right)} \times \\
& \times((\beta+2) \varepsilon)^{\frac{3 \beta}{2(\beta+2)}} \exp \left(-\frac{9}{2}((\beta+2) \varepsilon)^{-2}\right) .
\end{aligned}
$$




\section{Brownian local time}

Let $L_{t}^{x}(B)$ be the jointly continuous local time of a Brownian bridge $B$ at the point $x \in \mathbb{R}$ up to time $t \in[0,1]$.

M. Csörgő, Z. Shi, M. Yor, 1999: for any $m \in \mathbb{N}$,

$$
\int_{-\infty}^{\infty}\left(L_{1}^{x}(B)\right)^{m} d x \stackrel{\text { law }}{=} 2^{m-1} \int_{0}^{1}(\mathfrak{e}(t))^{m-1} d t .
$$

They also found the logarithmic asymptotics for

$$
\mathrm{P}\left\{\int_{-\infty}^{\infty}\left(L_{1}^{\times}(B)\right)^{2} d x \leq \varepsilon\right\} \text { and } \mathrm{P}\left\{\int_{-\infty}^{\infty}\left(L_{1}^{x}(B)\right)^{3} d x \leq \varepsilon\right\} .
$$




\section{Brownian local time}

Theorem 12. As $\varepsilon \rightarrow 0$

$$
\begin{gathered}
\mathrm{P}\left\{\int_{-\infty}^{\infty}\left(L_{1}^{x}(B)\right)^{3} d x \leq \varepsilon\right\} \sim \frac{8 \sqrt{6}}{\sqrt{\pi}} \varepsilon^{-1} \exp \left(-\frac{9}{2} \varepsilon^{-1}\right), \\
\mathrm{P}\left\{\int_{-\infty}^{\infty}\left(L_{1}^{x}(B)\right)^{2} d x \leq \varepsilon\right\} \sim \frac{8}{3} a_{1}^{3 / 2} \varepsilon^{-2} \exp \left(-\frac{8 a_{1}^{3}}{27 \varepsilon^{2}}\right),
\end{gathered}
$$

where $a_{1} \approx 2.3381$ is the absolute value of the first zero of the standard Airy function. 


\section{Brownian local time}

Let $T_{1}=\inf \{t: W(t)=1\}$ be the first hitting time of 1 by the Brownian motion. For $x \in[0,1]$ consider the local time process in $x$ up to the moment $T_{1}$ :

$$
Z(x)=L_{T_{1}}^{x}(W), \quad 0 \leq x \leq 1 .
$$

Theorem 13. Let $\psi$ be a regular-shaped weight on $[0,1]$. Then as $\varepsilon \rightarrow 0$

$$
\mathrm{P}\left\{\int_{0}^{1} L_{T_{1}}^{\times}(W) \psi(x) d x \leq \varepsilon^{2}\right\} \sim \frac{2 \sqrt{2} \psi^{1 / 4}(0)}{\sqrt{\pi} \vartheta \psi^{1 / 4}(1)} \varepsilon \exp \left(-\frac{\vartheta^{2}}{2} \varepsilon^{-2}\right) .
$$


More details and more results can be found in the paper

Nikitin, Ya. Yu. and Pusev, R. S. (2011). Exact $L_{2}$-small deviation asymptotics for some Brownian functionals. Preprint, 26 pp.

Available at http://arxiv.org/abs/1104.2891 